\title{
Novel amphiphilic carbon black composite nanoparticles from TEMPO-terminated polymer and TEMPO-terminated block copolymer grafted carbon black
}

\author{
Chia-Fen Lee ${ }^{\mathrm{a}}$, Cheng-Che Yang ${ }^{\mathrm{b}}$, Lee-Yih Wang ${ }^{\mathrm{c}}$, Wen-Yen Chiu ${ }^{\mathrm{b}, *}$ \\ ${ }^{a}$ Department of Cosmetic Science, Chia Nan University of Pharmacy and Science, Tainan, Taiwan, ROC \\ ${ }^{\mathrm{b}}$ Department of Chemical Engineering, National Taiwan University, Taipei, Taiwan, ROC \\ ${ }^{\mathrm{c}}$ Center for Condensed Matter Science, National Taiwan University, Taipei, Taiwan, ROC
}

Received 2 February 2005; received in revised form 24 April 2005; accepted 25 April 2005

Available online 26 May 2005

\begin{abstract}
The purpose of this study was to modify the surface characteristics of $\mathrm{CB}$ so as to prevent the aggregation of CB to provide the dispersibilities in either $\mathrm{H}_{2} \mathrm{O}$ or organic solvent. In this study, five kinds of hydrophilic TEMPO-terminated polymer, hydrophobic TEMPOterminated polymer and amphiphilic TEMPO-terminated block copolymer were synthesized. The five kinds of TEMPO-terminated polymers were: (1) poly(4-acetoxystyrene) (PAS-T), (2) poly(4-hydroxystyrene) (PHS-T), (3) polystyrene (PS-T), (4) poly(4-acetoxystyrene)-blockpolystyrene (PAS- $b$-PS-T), (5) poly(4-hydroxystyrene)-block-polystyrene (PHS- $b$-PS-T). These TEMPO-terminated polymers with desired molecular weights and specific structures were synthesized by using the method of living radical polymerization in the presence of 2,2,6,6tetramethylpiperidinyl-1-oxy (TEMPO). These TEMPO-terminated polymers and TEMPO-terminated block copolymers were grafted onto the surface of CB through a reaction of polymer radicals trapped by CB, so as to obtain the TEMPO-terminated polymer/CB and TEMPOterminated block copolymer/CB composite nanoparticles. Various variables such as reaction time, reaction temperature, amount of TEMPOterminated polymer, molecular weight of TEMPO-terminated polymer and amount of CB all of which influenced the grafting efficiency were investigated. Besides, the stability of the composite nanoparticles, which dispersed in $\mathrm{H}_{2} \mathrm{O}$ or organic solvent, was investigated by laser light scattering. The amphiphilic composite nanoparticles, PHS-T/CB and PHS- $b$-PS-T/CB, which dispersed well in both $\mathrm{H}_{2} \mathrm{O}$ and organic solvent, were synthesized successfully in this work.
\end{abstract}

(C) 2005 Elsevier Ltd. All rights reserved.

Keywords: Amphiphilic; Composite nanoparticles; Living radical polymerization

\section{Introduction}

Inorganic/organic composite material is widely applied in industry, while the disadvantages of the composite materials are that the inorganic particles are unable to disperse in organic phase uniformly so as to lower the mechanical strength of inorganic/organic composite material. Grafting of polymers onto the surface of inorganic

\footnotetext{
* Corresponding author. Address: Department of Materials Science and Engineering, National Taiwan University, Taipei 106, Taiwan, ROC. Tel./fax: +886223623259.

E-mail address: ycchiu@ccms.ntu.edu.tw (W.-Y. Chiu).
}

0032-3861/\$ - see front matter (C) 2005 Elsevier Ltd. All rights reserved. doi:10.1016/j.polymer.2005.04.053 particles was one of the most effective procedures to improve the surface properties of inorganic particles, so as to enhance the uniform of inorganic particles disperse in organic phase. Recently, surface-initiated grafted polymerization has been studied but it usually results in a poor control of chain-length and chains structure [1]. The techniques of living radical polymerization has proven very promising for the synthesis of low polydispersity linear polymers, block copolymers and star polymers [2-6]. Living polymerization techniques were successfully applied to surface-initiated graft polymerization to form the inorganic/organic composite material. Marutani et al. [7] reported the synthesis of magnetite nanoparticles coated with a well-defined graft polymer by using the method of living radical polymerization. In addition, Larnelle et al. [8] 
synthesized the inorganic/organic composite material consisting of poly( $n$-butyl acrylate)- $b$-poly(styrene) (PBA- $b$ PS) diblock copolymer anchored to silica particles via 'grafting from' technique using a living free radical polymerization. They pointed out that the morphology of the composite material was core/double shell structure. The materials obtained have different thermal behavior function of the ratio PBA/PS. Besides, in order to enhance the application of carbon black (CB) in industry, various kinds of polymers were grafted to the surfaces of CB. Sengupata et al. [9] reported that $N$-bromophthalimide (NBP) was a good thermal initiator and photo-initiator for free radical polymerization. They used NBP as the thermal initiator to proceed the radical graft polymerization of methyl methacrylate and acrylonitrile onto carbon black. The results of $T_{\mathrm{g}}$ measurements showed that $T_{\mathrm{g}}$ of the grafted carbon black was found to be higher than those of the corresponding homopolymers. Tsubokawa et al. reported the anionic, cationic, and radical graft polymerization of various monomers initiated by potassium carboxylate [10,11], acrylium [12] or benzylium perchlorate [13], and peroxyester [14] or azo [15] groups introduced onto carbon black, respectively. In the graft polymerization, the polymers were effectively grafted onto the surface by propagation of the grafted polymers from the surface of carbon black. In addition, Yoshikawa et al. [16] grafted polymers with controlled molecular weight and narrow molecular weight distribution onto carbon black surface by termination of living polymer cation with nucleophilic groups, such as amino groups and sodium phenolate and carboxylate groups, introduced onto the surface. The increase of molecular weight of the living polymer decreased the mole number of grafted polymer chains on carbon black surface due to the steric hindrance. Hayashi et al. [17] investigated the grafting reaction of peroxide polymers having pendant peroxycarbonate groups with carbon black surface. They pointed out that through heating of the peroxide
Table 1

Molecular weight and $T_{\mathrm{g}}$ of PAS-T polymers

\begin{tabular}{lrll}
\hline $\begin{array}{l}\text { Reaction time } \\
(\mathrm{h})\end{array}$ & $M_{\mathrm{n}}$ & $\begin{array}{l}\text { Polydispersity of } \\
\text { molecular weight }\end{array}$ & $T_{\mathrm{g}}\left({ }^{\circ} \mathrm{C}\right)$ \\
\hline 1.5 & 323 & 1.06 & Unseen \\
2.0 & 8485 & 1.23 & 117.9 \\
3.0 & 24,630 & 1.45 & 123.5 \\
\hline
\end{tabular}

polymers with carbon black in toluene, the corresponding polymers were grafted onto the surface. They suggested that polymer radicals formed by the thermal decomposition of pendant groups are trapped by polycondensed aromatic rings of carbon black.

In this study, TEMPO-terminated polymers, PAS-T, PS-T and TEMPO-terminated block copolymer, PAS- $b$-PS-T, were synthesized by using the method of living radical polymerization. Then these TEMPO-terminated polymers were grafted onto the surface of $\mathrm{CB}$ by a reaction of polymer radicals trapped by $\mathrm{CB}$ to form the PAS-T/CB, PS-T/CB and PAS- $b$-PS-T/CB composite nanoparticles. Various variables, which influenced the grafting efficiency, were investigated. In addition, $\mathrm{NH}_{4} \mathrm{OH}$ was used to proceed the hydrolysis reaction of PAS-T/CB and PAS- $b-\mathrm{PS}-\mathrm{T} / \mathrm{CB}$ to form the PHS-T/CB and PHS- $b$-PS-T/CB, respectively. The stability of the composite nanoparticles, which dispersed in $\mathrm{H}_{2} \mathrm{O}$ or organic solvents, was investigated.

\section{Experiment}

\subsection{Materials}

Styrene was distilled under a nitrogen atmosphere and reduced pressure prior to polymerization. Water was redistilled and deionized. The 2,2,6,6-tetramethylpiperidinyl-1-oxy (TEMPO), initiator (benzoyl peroxide (BPO)),

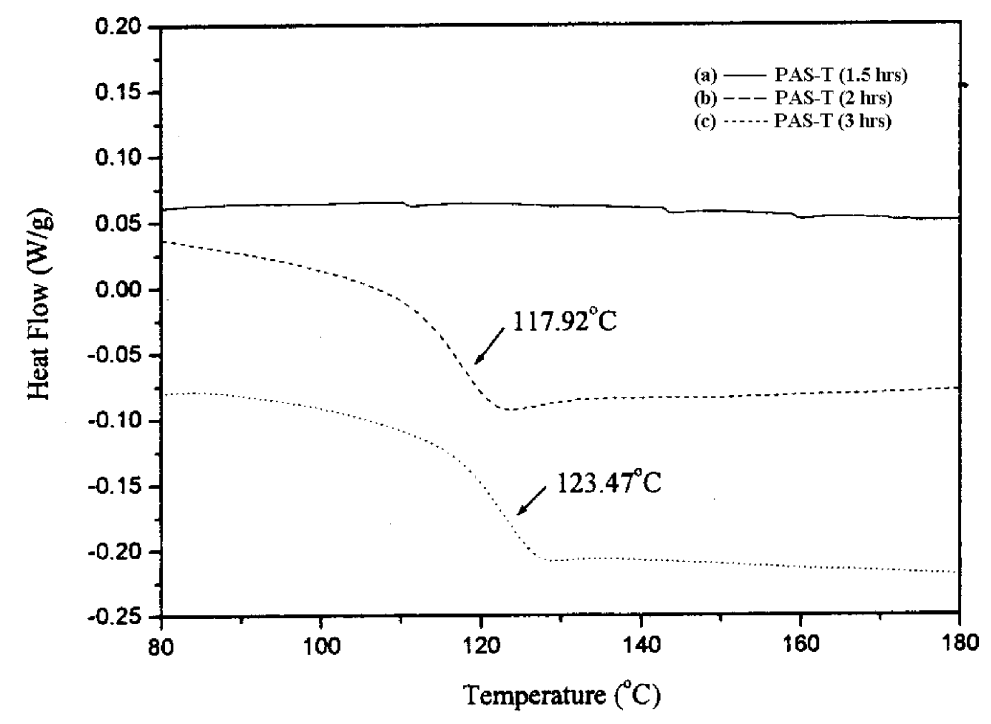

Fig. 1. Glass transition temperature of PAS-T after reacting for (a) $1.5 \mathrm{~h}$ (b) $2 \mathrm{~h}$ (c) $3 \mathrm{~h}$. 




Fig. 2. FTIR spectrograms of PAS-T and PHS-T TEMPO-terminated polymer.

4-acetoxystyrene (AS), styrene ( $\mathrm{St}$ ), and other chemicals were of analytical grade and used without further purification. The carbon black (CB) used was Monarch 900 (CABOT Co., Ltd). The average diameter of CB was about $15 \mathrm{~nm}$.

\subsection{Synthesis of PAS-T and PS-T TEMPO-terminated polymers and PAS-b-PSt-T TEMPO-terminated block copolymers}

TEMPO $(0.018 \mathrm{M}), \mathrm{BPO}(0.015 \mathrm{M})$ and AS $(5 \mathrm{ml})$ or (styrene $(5 \mathrm{ml})$ ) were charged into a reactor, and then the reaction mixture was pre-heated for $3.5 \mathrm{~h}$ at the temperature of $95{ }^{\circ} \mathrm{C}$. Afterwards, the temperature was raised to $125^{\circ} \mathrm{C}$ to synthesize the TEMPO-terminated polymer PAS-T (or PS-T). After the polymerization reaction, PAS-T (or PS-T) was obtained as a precipitate from a large excess of methanol, purified by centrifuge with methanol in order to remove the residual AS (or styrene) monomer. Afterwards, PAS-T $(30 \mu \mathrm{mol})$ was used as the macro initiator to polymerize styrene $(2 \mathrm{ml})$ at the temperature of $125^{\circ} \mathrm{C}$ to form the TEMPO-terminated PAS- $b$-PSt-T copolymer.

$\mathrm{NH}_{4} \mathrm{OH}(10 \mathrm{ml})$ was used to proceed the hydrolysis reaction of PAS-T and PAS- $b$-PS-T for $24 \mathrm{~h}$ at the temperature of $25^{\circ} \mathrm{C}$ to form the PHS-T hydrophilic TEMPO-terminated polymer and PHS- $b$-PS-T amphiphilic TEMPO-terminated copolymer, respectively. The PHS-T and PHS- $b$-PS-T was purified by centrifuge with $\mathrm{H}_{2} \mathrm{O}$ to remove the residual $\mathrm{NH}_{4} \mathrm{OH}$.

\subsection{Molecular weight and molecular weight distribution of TEMPO-terminated polymers}

The molecular weight and molecular weight distribution of TEMPO-terminated polymers were measured by using the gel permeation chromatography, GPC (Showa Denko kk, model: Showa RI-71).

\subsection{Synthesis of TEMPO-terminated polymers/CB and TEMPO-terminated block copolymer/CB composite particles}

TEMPO-terminated polymer (PAS-T or PS-T) $(30 \mu \mathrm{mol})$ or TEMPO-terminated block copolymer (PAS- $b$-PS-T) (30 $\mu \mathrm{mol}), \mathrm{CB}(0.05 \mathrm{~g})$, and $N, N$-dimethylformamide (DMF) $(20 \mathrm{ml})$ were charged into a reactor. Then the reaction mixture was heated under stirring at $125^{\circ} \mathrm{C}$, and reacted for $12 \mathrm{~h}$. The TEMPO-terminated polymers or

Table 2

The molecular weight and $T_{\mathrm{g}}$ of PAS- $b$-Pst-T copolymer ${ }^{\circ} \mathrm{C}$

\begin{tabular}{|c|c|c|c|c|}
\hline $\begin{array}{l}\text { Molecular weight of } \\
\text { PAS-T }\end{array}$ & $\begin{array}{l}\text { Reaction time }(\mathrm{h}) \text { for the synthesis of PAS- } b \text { - } \\
\text { PSt-T using PAS-T as macroinitiator }\end{array}$ & $\begin{array}{l}\text { Molecular weight of } \\
\text { PAS- } b \text {-PSt-T }\end{array}$ & $T_{\mathrm{g}}\left({ }^{\circ} \mathrm{C}\right)$ of PAS- $b$-PSt-T & \\
\hline \multirow[t]{3}{*}{8485} & 0.5 & 7729 & PAS; PSt & 115.8; unseen \\
\hline & 1 & 12,680 & PAS; PSt & $117.8 ; 101.0$ \\
\hline & 2 & 17,330 & PAS; PSt & $121.3 ; 106.1$ \\
\hline 24,630 & 1 & 40,360 & & \\
\hline
\end{tabular}




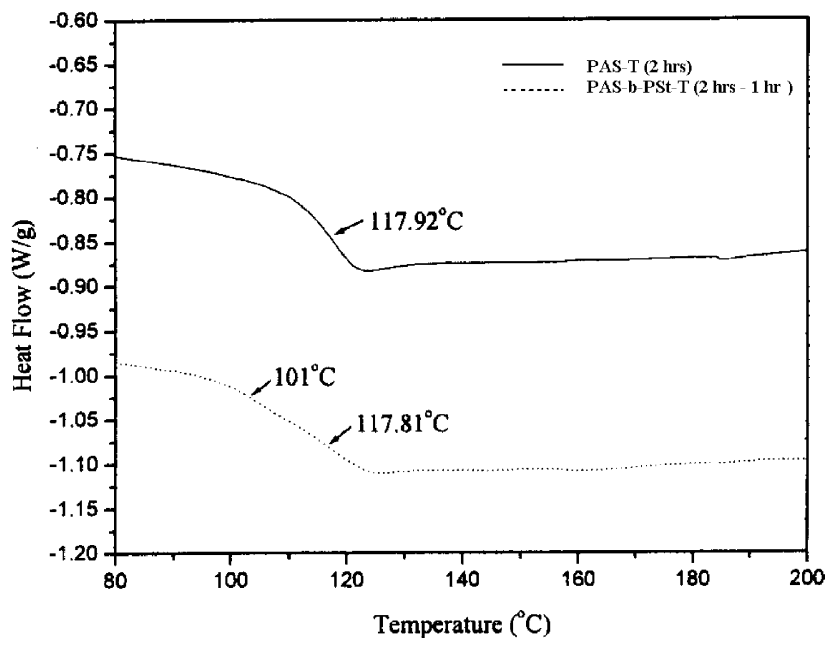

Fig. 3. The DSC curves of PAS-T and PAS- $b$-PSt-T copolymers.

TEMPO-terminated block copolymer were grafted onto the CB surface through the trapping of polymer radicals by carbon black surface to form the TEMPO-terminated polymer/CB composite particles (PAS-T/CB or PS-T/CB) or TEMPO-terminated block copolymer/CB composite particles (PAS- $b$-PS-T/CB). After the reaction, the reaction mixture was poured into a large excess of methanol to precipitate the TEMPO-terminated polymer/CB composite particle or TEMPO-terminated block copolymer/CB composite particles. In order to remove the ungrafted TEMPOterminated polymer, the TEMPO-terminated polymer/CB composite particles were dispersed in THF and the dispersive solution was centrifuged. This procedure was repeated until no more TEMPO-terminated polymer could be detected in the supernatant solution.

Besides, $\mathrm{NH}_{4} \mathrm{OH}(10 \mathrm{ml})$ was used to proceed the hydrolysis reaction of PAS-T/CB and PAS- $b-\mathrm{PS}-\mathrm{T} / \mathrm{CB}$ composite particles for $24 \mathrm{~h}$ to form the PHS-T/CB and PHS- $b$-PS-T/CB composite particles, respectively, at the temperature of $25^{\circ} \mathrm{C}$. These composite particles were purified by centrifuge with $\mathrm{H}_{2} \mathrm{O}$ to remove the residual $\mathrm{NH}_{4} \mathrm{OH}$, and then the composite particles were dried under the vacuum condition at the temperature of $50{ }^{\circ} \mathrm{C}$.

Table 3

Relationship between the molecular weight of PAS and the efficiency of PHS-T grafted onto the surface of CB

\begin{tabular}{lccc}
\hline $\begin{array}{l}\text { Reaction } \\
\text { time (h) }\end{array}$ & $\begin{array}{l}\text { Molecular weight } \\
\text { of PAS }\end{array}$ & $\begin{array}{l}\text { Weight } \\
\text { percentage of } \\
\text { grafting }(\%)\end{array}$ & $\begin{array}{l}\text { Mole number of } \\
\text { grafting }\left(G_{\mathrm{n}}\right)\end{array}$ \\
\hline 1.5 & 323 & 5.50 & 170.34 \\
2.0 & 8485 & 13.70 & 19.96 \\
3.0 & 24,630 & 19.52 & 7.92 \\
\hline
\end{tabular}

Reaction temperature, $125^{\circ} \mathrm{C}$; mole of PAS-T, $30 \mu \mathrm{mol}$; volume of DMF, $20 \mathrm{ml}$; weight of $\mathrm{CB}, 0.05 \mathrm{~g}$; grafting time, $12 \mathrm{~h}$.
Table 4

Relationship between reaction time of grafting and weight percentage of PS-T grafted onto the surface of CB

\begin{tabular}{ll}
\hline Reaction time $(\mathrm{h})$ & Percentage of grafting $(\%)$ \\
\hline 3 & 10.87 \\
6 & 11.91 \\
12 & 13.08 \\
18 & 14.25 \\
24 & 14.47
\end{tabular}

Reaction temperature, $125^{\circ} \mathrm{C}$; weight of PS-T, $0.19 \mathrm{~g}$; weight of $\mathrm{CB}, 0$. $05 \mathrm{~g}$; volume of DMF, $20 \mathrm{ml}$; molecular weight of PS-T, 5610.

Table 5

Relationship between reaction temperature and weight percentage of PS-T grafted onto the surface of $\mathrm{CB}$

\begin{tabular}{lc}
\hline Reaction temperature $\left({ }^{\circ} \mathrm{C}\right)$ & Percentage of grafting $(\%)$ \\
\hline 25 & 5.19 \\
115 & 9.81 \\
125 & 13.08 \\
135 & 13.69 \\
\hline
\end{tabular}

Reaction time, $12 \mathrm{~h}$; weight of PS-T, $0.19 \mathrm{~g}$; weight of $\mathrm{CB}, 0.05 \mathrm{~g}$; volume of DMF, $20 \mathrm{ml}$; molecular weight of PS-T, 5610.

Table 6

Relationship between the weight of PS-T and weight percentage of PS-T grafted onto the surface of $\mathrm{CB}$

\begin{tabular}{ll}
\hline Weight of PS-T $(\mathrm{g})$ & Percentage of grafting $(\%)$ \\
\hline 0.10 & 11.90 \\
0.19 & 13.08 \\
0.30 & 15.80
\end{tabular}

Reaction time of grafting, $12 \mathrm{~h}$; reaction temperature, $125^{\circ} \mathrm{C}$; weight of $\mathrm{CB}, 0.05 \mathrm{~g}$; volume of DMF, $20 \mathrm{ml}$; molecular weight of PS-T, 5610.

Table 7

The weight percentage of PAS- $b$-PSt-T copolymer grafted onto the surface of $\mathrm{CB}$

\begin{tabular}{ll}
\hline $\begin{array}{l}\text { Molecular weight of } \\
\text { PAS- } b \text {-PSt-T }\end{array}$ & $\begin{array}{l}\text { Weight percentage of PAS- } b \text {-PSt-T grafted } \\
\text { onto the surface of CB }(\%)\end{array}$ \\
\hline 7729 & 6.71 \\
12,680 & 32.38 \\
17,330 & 12.04 \\
\hline
\end{tabular}

The molecular weight of the macroinitiator PAS-T is 8485 .

\subsection{Measurement of grafting efficiency}

The polymer, which was grafted onto the surface of $\mathrm{CB}$, was degradable after isothermal heating for $30 \mathrm{~min}$ at the temperature of $500{ }^{\circ} \mathrm{C}$ by a thermal analyzer (TGA). The carbon black (CB) showed a weight loss of $3.55 \%$. The weight percentage of grafting and mole number of grafted polymer chains onto carbon black were calculated by the 




Fig. 4. The TEM photograph of carbon black.

following equations:

Grafting wt $\%=\left[\frac{\left(W_{\mathrm{p}}-W_{\mathrm{B}}\right)}{W_{\mathrm{p}}}\right] 100 \%$

Grafting mol $\%=\left(\frac{\mu \mathrm{mol}}{g-\text { carbon black }}\right)$

$$
=\frac{\left[\left(W_{\mathrm{p}}-W_{\mathrm{B}}\right) / M_{\mathrm{n}}\right] \times 10^{6}}{W_{\mathrm{p}}}
$$

where $W_{\mathrm{p}}$ is the residual weight of $\mathrm{CB}$ under the isothermal heating on TGA. $W_{\mathrm{B}}$ is the residual weight of TEMPOterminated polymer/CB composite particles under the isothermal heating on TGA. $M_{\mathrm{n}}$ is the average molecular weight of TEMPO-terminated polymer.

\subsection{Stability of the dispersive solution of TEMPO-} terminated polymer/CB and TEMPO-terminated block copolymer/CB composite particles

The TEMPO-terminated polymer/CB or TEMPO-terminated block copolymer/CB composite particles were dispersed in either THF or $\mathrm{H}_{2} \mathrm{O}$ to form the dispersive solution, and then rested for a period of time to measure the variance of the particles size of composite particles so as to identify the stability of the dispersive solution.

\subsection{Morphology of $C B$ and TEMPO-terminated polymer/CB composite particles}

The morphologies of CB particles and TEMPO-terminated polymer/CB composite particles were observed by using a JSM-1200 EXII transmitting electron microscope (TEM).

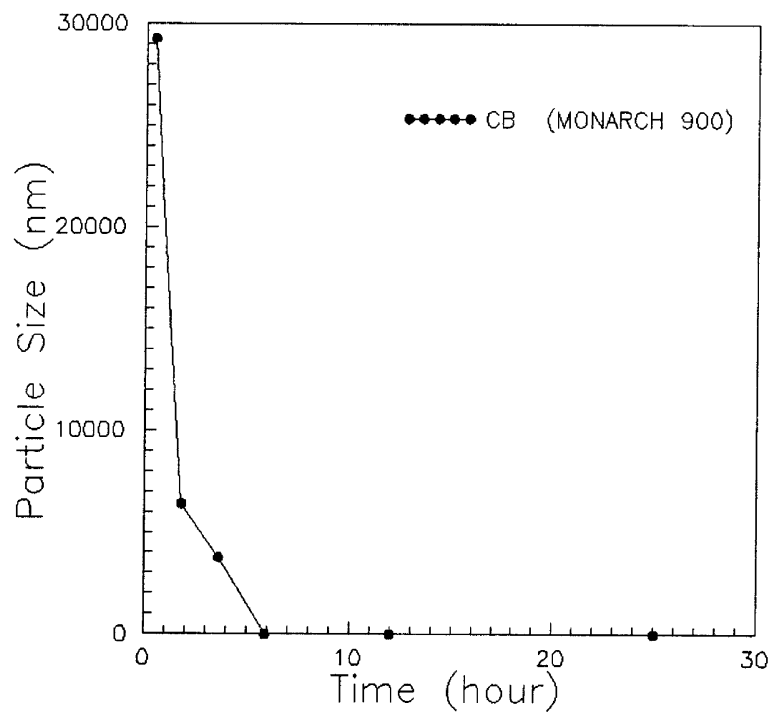

Fig. 5. The sizes of $\mathrm{CB}$ particles which dispersed in $\mathrm{H}_{2} \mathrm{O}$, measured by laser light scattering.

\section{8. $T_{g}$ of TEMPO-terminated polymers}

The $T_{\mathrm{g}}$ of TEMPO-terminated polymers or TEMPOterminated block copolymer was measured by differential scanning calorimetry, DSC (TA Instrument, model: DSC 2010). The temperature was increased by the rate of $20{ }^{\circ} \mathrm{C} / \mathrm{min}$.

\section{Results and discussion}

\subsection{Synthesis of PAS-T and PHS-T TEMPO-terminated polymer}

Table 1 showed the molecular weight and molecular weight distribution of PAS-T obtained from the living radical polymerization. It was obvious that the molecular weight of PAS-T increased with increasing reaction time. After reacting for $1.5 \mathrm{~h}$, the molecular weight of PAS was 323 , however, the molecular weight of PAS increased to 24,630 after reacting for $3 \mathrm{~h}$. For the course of reaction, the polydispersity of PAS-T was lower than 1.5. In addition, the increase of reaction time increased the glass transition temperature $\left(T_{\mathrm{g}}\right)$ of PAS-T as shown in Table 1 and Fig. 1, corresponding to the increasing molecular weight of polymers. This result indicated that TEMPO-terminated PAS was a living polymer, which was successfully synthesized by the method of living radical polymerization. Furthermore, the PAS-T was hydrolyzed by $\mathrm{NH}_{4} \mathrm{OH}$ to form the PHS-T. The FTIR spectrogram of PAS-T showed the significant peak of $\mathrm{C}=\mathrm{O}$ group $\left(1762.16 \mathrm{~cm}^{-1}\right)$ as shown in Fig. 2. After the PAS-T was hydrolyzed by $\mathrm{NH}_{4} \mathrm{OH}$, the peak of $\mathrm{C}=\mathrm{O}$ group disappeared, and showed the significant peak of OH group. $T_{\mathrm{g}}$ of PHS-T was about $170-190{ }^{\circ} \mathrm{C}$. 


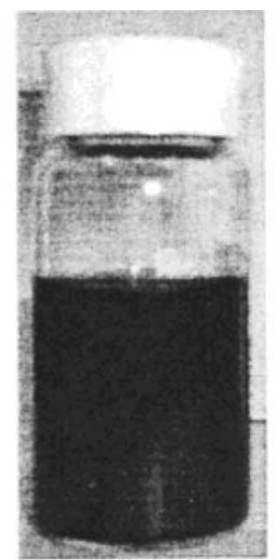

$\mathbf{t}=\mathbf{0} \min$

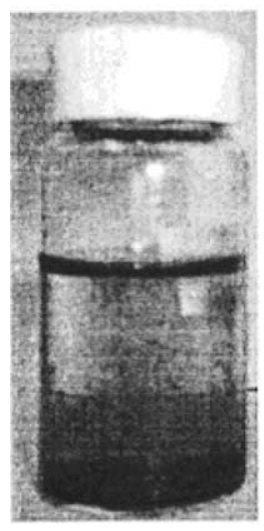

$\mathbf{t}=\mathbf{2}$ hrs

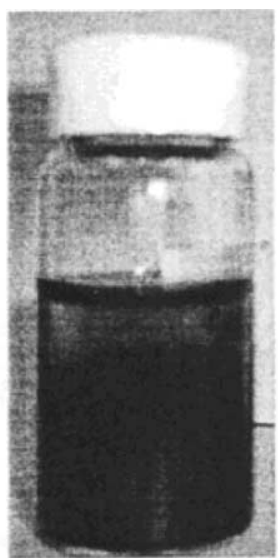

$\mathbf{t}=30 \mathrm{~min}$

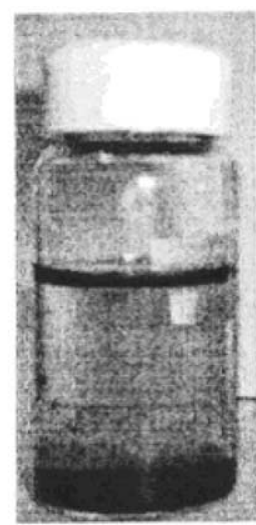

$\mathbf{t}=\mathbf{3}$ hrs

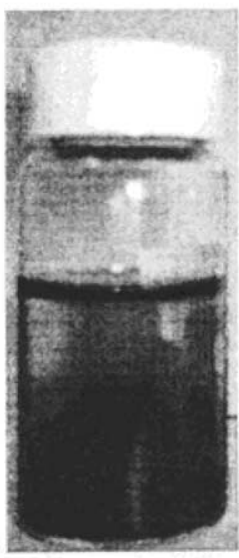

$\mathbf{t}=\mathbf{1} \mathbf{l l} \mathbf{x}$

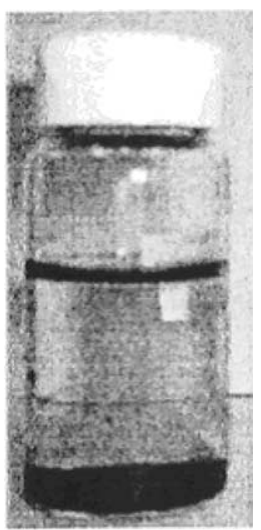

$\mathrm{t}=6 \mathrm{hrs}$

Fig. 6. $\mathrm{CB}$ particles dispersed in $\mathrm{H}_{2} \mathrm{O}$.

\subsection{Synthesis of PAS-b-PSt-T TEMPO-terminated block copolymers}

PAS-T was used as the macro initiator to polymerize styrene at the temperature of $125^{\circ} \mathrm{C}$ to form PAS- $b$-PSt-T block copolymer. Table 2 showed the effect of reaction time on the molecular weight and $T_{\mathrm{g}} \mathrm{s}$ of PAS- $b$-PSt-T TEMPOterminated copolymer. After reacting for $0.5 \mathrm{~h}$, the molecular weight of PAS- $b$-PSt-T copolymer did not increase significantly, and the DSC curve did not show the $T_{\mathrm{g}}$ of polystyrene. It indicated that the styrene block in the PAS- $b$ PSt-T copolymer was not long enough to be detectable after

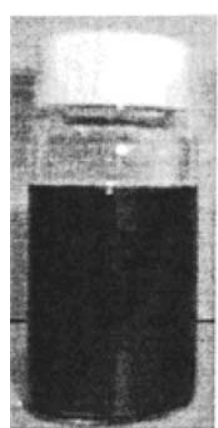

Day 1



Day 2

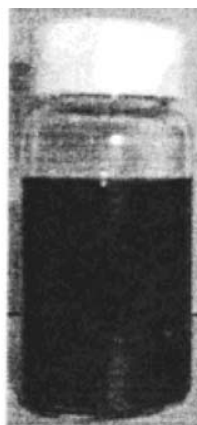

Day 3

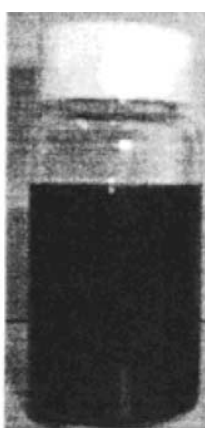

Day 4

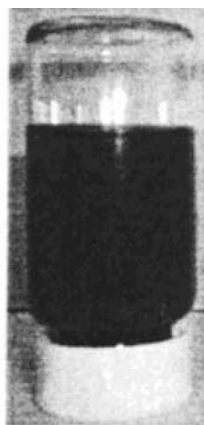

Day 5

Fig. 7. PAS-T/CB composite particles colloidal dispersion in THF. 


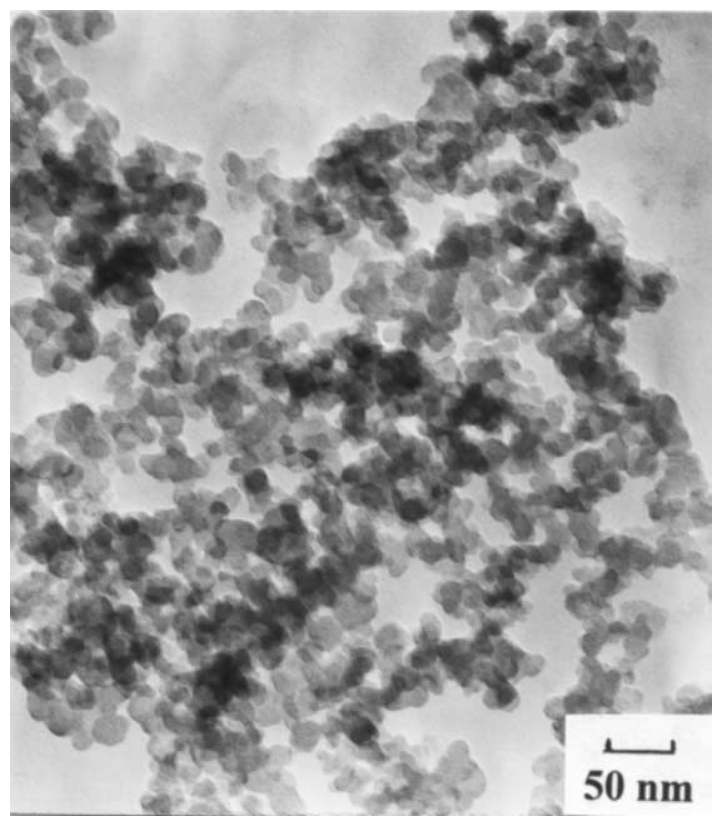

Fig. 8. TEM photograph of PHS-T/CB composite particles.

reacting for $0.5 \mathrm{~h}$. With increasing the reaction time, the molecular weight of PAS- $b$-PSt-T copolymer increased as shown in Table 2, and the $T_{\mathrm{g}}$ of PS block was then clearly seen in the DSC curve of Fig. 3, where the $T_{\mathrm{g}}$ of PAS block was about $118^{\circ} \mathrm{C}$ and the $T_{\mathrm{g}}$ of PS block was about $101{ }^{\circ} \mathrm{C}$. This meant that during polymerization, the PS block in PAS- $b$-PSt-T copolymer was growing in molecular weight gradually. That is PAS- $b$-PSt-T was a living copolymer. In addition, the molecular weight of the macro-initiator, PAS$\mathrm{T}$, influenced the molecular weight growth of PAS- $b$-PSt-T significantly. Table 2 showed that when a large molecular weight of PAS was used as the macro-initiator, the molecular weight of PAS- $b$-PSt-T thus obtained was higher.

\subsection{Synthesis of TEMPO-terminated polymer/CB composite particles}

In order to modify the surface characteristics of $\mathrm{CB}$, the PAS-T/CB, PHS-T/CB and PS-T/CB composite particles were synthesized. The effect of molecular weight of PAS on the percentage of PHS-T grafted onto the surface of CB was shown in Table 3. The increase of molecular weight of PAS$\mathrm{T}$ increased the weight percentage of grafting but decreased the mole number of PHS-T chains $\left(G_{\mathrm{n}}\right)$ grafted onto CB. The PAS-T with a larger molecular weight was more difficult to graft onto the surface of $\mathrm{CB}$ due to the steric hindrance. Hence, although the grafted number of polymer chains decreased, the weight percentage of grafting may still be higher because the grafted polymer chains had a larger molecular weight.

In addition, PS-T polymer was synthesized and then grafted onto the surface of $\mathrm{CB}$. Table 4 showed the percentage of PS-T grafting onto $\mathrm{CB}$ at different reaction times. The percentage of grafting increased with increasing the reaction time and gradually leveled off after $18 \mathrm{~h}$. The effect of reaction temperature on the grafting reaction of PS$\mathrm{T}$ with $\mathrm{CB}$ was shown in Table 5. It was found that the percentage of PS-T grafting onto the surface of $\mathrm{CB}$ increased with the increase of temperature, due to the fact that the mediated TEMPO was easier to escape away from the chain ends of the polymers at higher temperatures, which increased the opportunity of polymer radicals to react with $\mathrm{CB}$, and increased the percentage of PS-T grafted onto the surface of CB. In addition, the increase of the amount of PS-T increased the opportunity of PS-T to react with CB, so

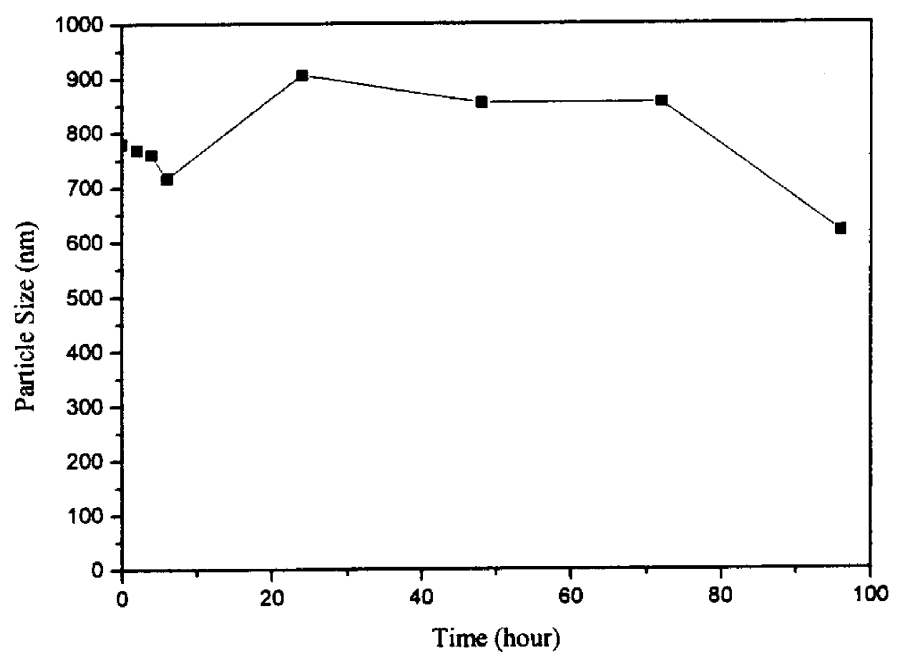

Fig. 9. The particle sizes of PHS-T/CB composite particles dispersed in $\mathrm{H}_{2} \mathrm{O}$, measured by laser light scattering. 


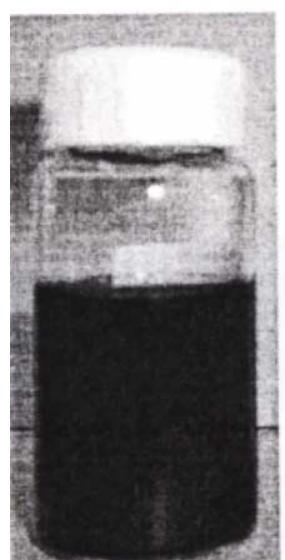

Dayl

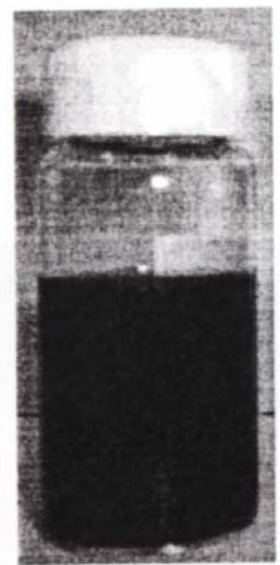

Day2

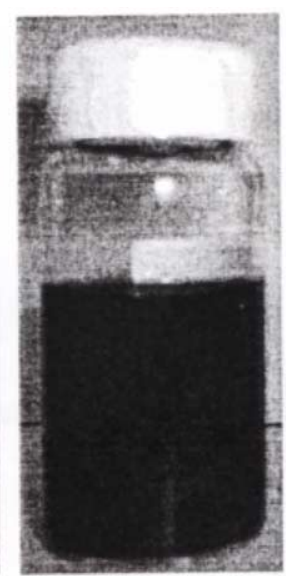

Day3

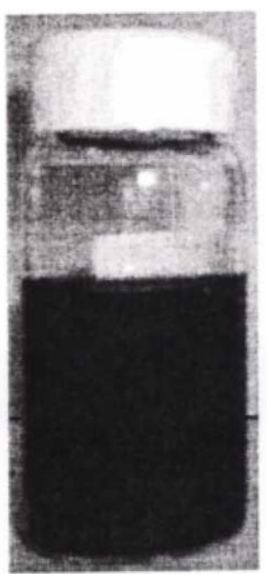

Day4

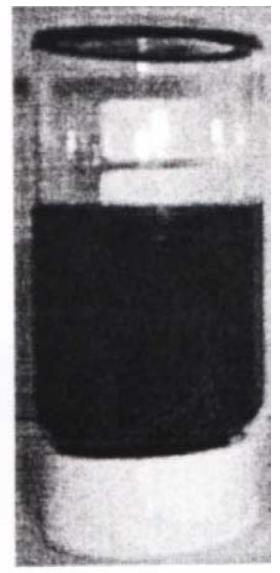

Day5

Fig. 10. PHS-T/CB colloidal dispersion in $\mathrm{H}_{2} \mathrm{O}$.

as to increase the percentage of PS-T grafted onto the surface of $\mathrm{CB}$ as shown in Table 6.

\subsection{Synthesis of TEMPO-terminated block copolymer/CB composite particles}

PAS- $b$-PSt-T TEMPO-terminated block polymer was synthesized and then grafted onto the surface of carbon black. The molecular weight of PAS- $b$-PSt-T copolymer influenced the weight percentage of grafting significantly. Table 7 showed the relationship between the molecular weight of PAS- $b$-PSt-T copolymer and the weight percentage of PAS- $b$-PSt-T copolymer grafted onto the surface of carbon black. The results showed that the PAS- $b$-PSt-T with a moderate molecular weight of 12,680 had the highest grafting percentage compared to that of smaller or larger molecular weights, 7729 or 17,330. Two factors, polymer chain length and steric hindrance, competed to influence the weight percentage of grafting.

\subsection{Dispersibility of $C B$}

Fig. 4 showed the TEM photograph of carbon black, the diameter of $\mathrm{CB}$ was about $15 \mathrm{~nm}$. In a dispersibility experiment, the carbon black was forced to disperse in $\mathrm{H}_{2} \mathrm{O}$ and they rested for observation. Those results were shown as Fig. 5. At the initial measurement, the diameter of carbon black dispersed in $\mathrm{H}_{2} \mathrm{O}$ was large (about $30 \mu \mathrm{m}$ ) due to the serious coagulation of carbon black. After the dispersive solution was rested for 2 and $4 \mathrm{~h}$, the large particles of carbon black had precipitated to the bottom, and only small particles dispersed in the aqueous medium were detected. After being rested for $6 \mathrm{~h}$, all particles coagulated and precipitated to the bottom, so the laser light scattering was unable to detect any $\mathrm{CB}$ particles in $\mathrm{H}_{2} \mathrm{O}$. Also, as shown in Fig. 6, all of the carbon black precipitated to the bottom after the dispersive solution was rested for $6 \mathrm{~h}$. A similar result was observed for the dispersion solution of $\mathrm{CB}$ in THF. That meant the unmodified $\mathrm{CB}$ was unable to disperse well in THF or $\mathrm{H}_{2} \mathrm{O}$.

\subsection{Dispersibility of TEMPO-terminated polymer/CB composite particles}

The dispersibility of $\mathrm{CB}$ was remarkably improved by the grafting of polymers onto the surfaces. The PAS-T/CB composite particles colloidal dispersion in THF was stable as shown in Fig. 7. Also, PHS-T/CB and PS-T/CB composite particles were able to disperse in THF (not shown). Besides, the stability of PHS-T/CB, PAS-T/CB, and PS-T/CB colloidal dispersion in $\mathrm{H}_{2} \mathrm{O}$ was investigated. It was found that both PS-T/CB and PAS-T/CB composite particles were unable to disperse in $\mathrm{H}_{2} \mathrm{O}$, but PHS-T/CB particles were well dispersed in $\mathrm{H}_{2} \mathrm{O}$ due to the hydrophilic

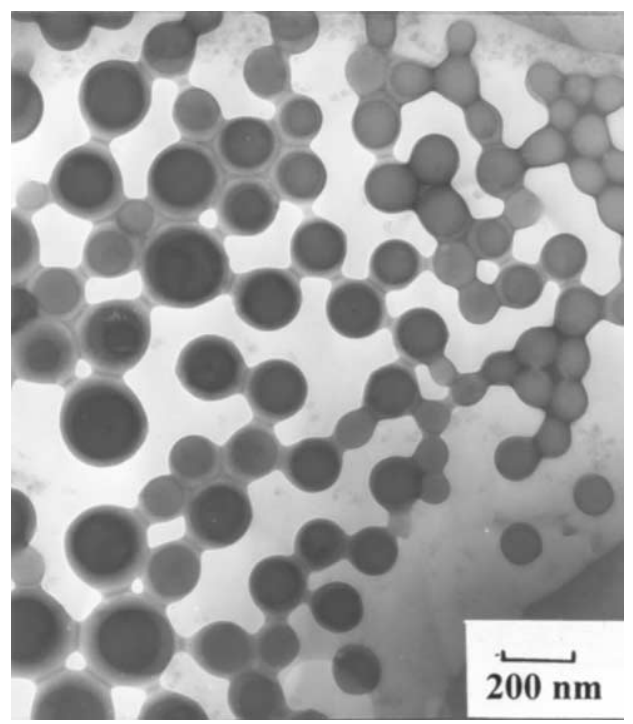

Fig. 11. The TEM photograph of PAS- $b$-PSt-T/CB composite particles. 


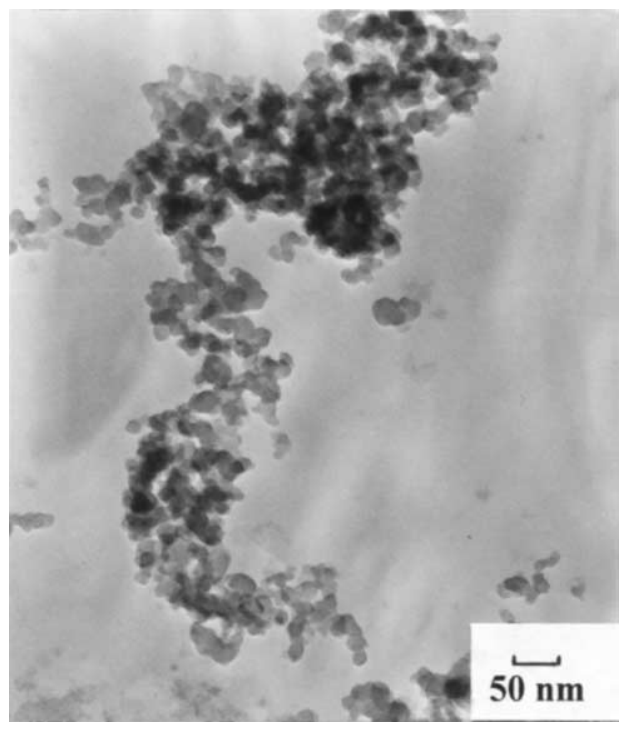

Fig. 12. The TEM photograph of the PHS- $b$-PSt-T/CB composite particles.

nature of PHS-T. Fig. 8 showed the TEM photograph of PHS-T/CB composite particles, which had a uniform particle size and the diameter was about $25 \mathrm{~nm}$. Fig. 9 showed the particle sizes of PHS-T/CB composite particles dispersed in $\mathrm{H}_{2} \mathrm{O}$, measured by laser light scattering. During the time of measurement, the particle sizes did not change much. Also, the PHS-T/CB colloidal dispersion in $\mathrm{H}_{2} \mathrm{O}$ was observed as Fig. 10. Both Figs. 9 and 10 indicated that the dispersion of the PHS-T/CB particles in water was quite stable. That is, PHS-T/CB composite particles had amphiphilic properties, which enabled them to disperse well in either organic solvent or $\mathrm{H}_{2} \mathrm{O}$.

\subsection{Dispersibility of TEMPO-terminated block copolymer/CB composite particles}

After the grafting of PAS- $b$-PSt-T TEMPO-terminated copolymers onto the surfaces of $\mathrm{CB}$, the composite particles were forced to disperse in $\mathrm{H}_{2} \mathrm{O}$ ready for the TEM observation. The TEM photograph of PAS- $b$-PSt-T/CB composite particles was shown as Fig. 11. It showed that the carbon black particles were encapsulated completely by PAS- $b$-PSt-T copolymer and the size was about 100 $250 \mathrm{~nm}$. Through similar dispersibility experiments as above, the PAS- $b$-PSt-T/CB composite particles were found to be able to disperse well in THF, but unable to disperse well in $\mathrm{H}_{2} \mathrm{O}$, due to the hydrophobic property of PAS- $b$-PSt-T copolymer.

In addition, the PAS- $b$-PSt-T/CB composite particles were hydrolyzed to form the amphiphilic block copolymer PHS- $b$-PSt-T/CB composite particles. Fig. 12 showed the TEM photograph of the PHS- $b$-PSt-T/CB composite particles. The particle size was uniformly about $25 \mathrm{~nm}$. Under the detection of laser light scattering, the size of PHS$b$-PSt-T/CB composite particles dispersed in $\mathrm{H}_{2} \mathrm{O}$ was about $120 \mathrm{~nm}$, invariant with time as seen in Fig. 13. It indicated that the PHS- $b$-PSt-T/CB composite particles were able to disperse well in $\mathrm{H}_{2} \mathrm{O}$. The sizes of the composite particles measured by laser light scattering were larger than that observed by TEM. Because in the measurement of laser light scattering, the composite particles were in a dispersive state in solution, in which the hydrophilic (or hydrophobic) segments of PHS- $b$-PSt-T fully extended in $\mathrm{H}_{2} \mathrm{O}$ (or THF) so that the particle size was significantly enlarged. Similar experiments were done for PHS- $b$-PSt-T/CB composite particles, which were dispersed in THF, and stability of the dispersive solution evidenced that the PHS- $b$-PSt-T/CB composite particles were also able to disperse well in THF.

\section{Conclusion}

Carbon black is widely used in industry while the capability of the carbon black is restricted by the

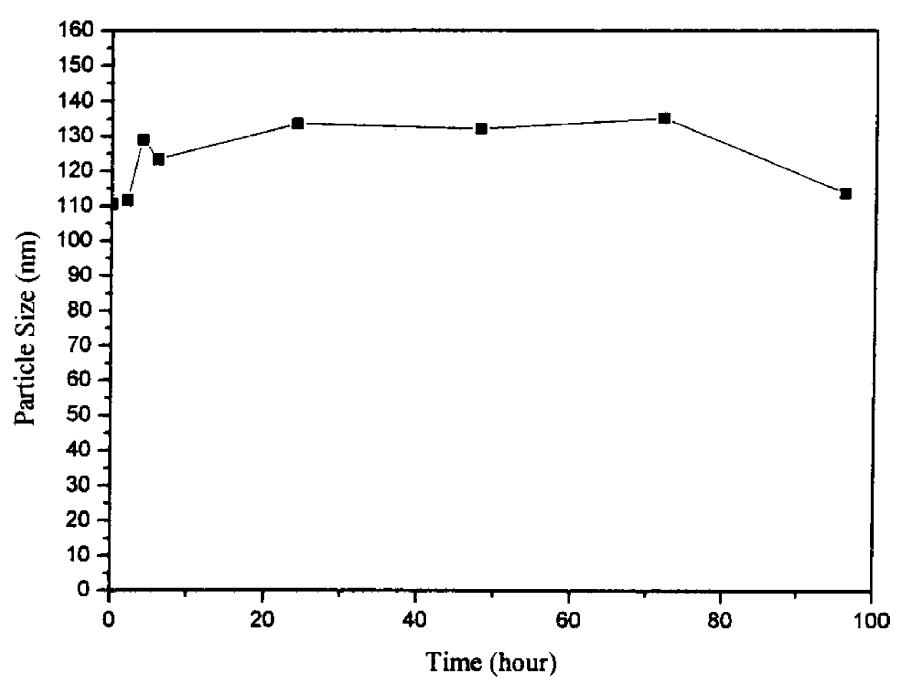

Fig. 13. The sizes of PHS- $b$-PSt-T/CB composite particles which dispersed in $\mathrm{H}_{2} \mathrm{O}$, measured by laser light scattering. 
disadvantage of difficult to disperse well in $\mathrm{H}_{2} \mathrm{O}$ or organic solvent. In this study, the TEMPO-terminated polymer and TEMPO-terminated block copolymer were grafted onto the carbon black to modify the characteristics of the surface of carbon black successfully. Various variables such as molecular weight of TEMPO-terminated polymer, reaction time and reaction temperature, influenced the percentage of grafting significantly. PS-T/CB, PAS-T/CB and PAS- $b$-PS-T/CB hydrophobic composite particles dispersed well in THF. Amphiphilic PHS-T/CB and PHS- $b$-PS-T/CB composite particles dispersed well in both THF and $\mathrm{H}_{2} \mathrm{O}$.

\section{References}

[1] Boven G, Oosterling MLCM, Challa G, Schouten AJ. Polymer 1990; 45:2231.

[2] Haruyuki O, Yusuke T, Masahiro T, Masamitsu S. Polymer 2002;43: 3155.
[3] Gabaston LI, Furlong SA, Jackson RA, Arms SP. Polymer 1999;40: 4505.

[4] Astsushi N, Takeshi M, Harumi K, Toshifumi S, Toyoji K. Polymer 2002;43:4835.

[5] Shim SE, Oh S, Chang YH, Jin MJ, Choe S. Polymer 2004;45: 4731.

[6] Abrol S, Caulfield MJ, Qiao GG, Solomon DH. Polymer 2001;42: 5987.

[7] Marutani E, Yamamoto S, Ninjbadgar T, Tsujii Y, Fukuda T, Takano M. Polymer 2004;45:2231.

[8] Laruelle G, Parvole J, Francois J, Billon L. Polymer 2004;45:5013.

[9] Sengupta PK, Pramanik AR. Eur Polym J 1994;30:421.

[10] Tsubokawa N, Funaki A, Hada Y, Sone Y. J Polym Sci, Polym Chem Ed 1987;20:3297.

[11] Tsubokawa N, Yamada A, Sone Y. Polym Bull 1983;10:62.

[12] Tsubokawa N. J Polym Sci, Polym Chem Ed 1983;21:705.

[13] Tsubokawa N, Handa S. J Macromol Sci, Pure Appl Chem A 1993;30: 277.

[14] Tsubokawa N, Fujiki K, Sone Y. Polym J 1988;20:213.

[15] Fujki K, Tsubokawa N, Sone Y. Polym J 1990;22:661.

[16] Yoshikawa S, Tsubokawa N. Polym J 1996;28:317.

[17] Hayashi S, Handa S, Oshibe Y, Yamamoto T, Tsubokawa N. Polym J 1995;27:623. 